(2) Open Access Full Text Article

\title{
Long-term progression-free survival of apatinib monotherapy for relapsed ovarian cancer: a case report and literature review
}

This article was published in the following Dove Press journal:

OncoTargets and Therapy

\section{Di Zhang ${ }^{1,2}$ \\ Jiaqi Huang ${ }^{1,2}$ \\ Yulan Sun ${ }^{2}$ \\ Qisen Guo'}

'Shandong Cancer Hospital Affiliated to Shandong University, Shandong University, Jinan 250II7, People's Republic of China; ${ }^{2}$ Shandong Academy of Medical Sciences, Shandong Cancer Hospital and Institute, Shandong Cancer Hospital affiliated to Shandong University, Jinan 250II7, People's Republic of China

\begin{abstract}
Ovarian cancer is the deadliest gynecologic malignancy, which poses a great threat to female health. Anti-angiogenic therapy could bring clinical benefit for patients with ovarian cancer. Apatinib, an oral small-molecule vascular endothelial growth factor receptor2 inhibitor, has shown notable therapeutic effect in a wide variety of tumors. We report a woman with advanced ovarian cancer who received apatinib at $250 \mathrm{mg} /$ day after failure of multiple-line treatment regimens, followed by discussion through review of literature. The patient has quite a long progression-free survival time of 24 months, with a satisfactory quality of life. Apatinib monotherapy may provide an additional option for advanced ovarian cancer, but it still needs further observation and exploration.
\end{abstract}

Keywords: ovarian cancer, apatinib monotherapy, anti-angiogenic therapy, vascular endothelial growth factor receptor

\section{Introduction}

Ovarian cancer, a female reproductive system malignancy, is the leading cause of gynecologic cancer deaths. In 2018, approximately 22,240 patients were diagnosed with ovarian cancer and 14,070 deaths were connected with this disease in the United States. ${ }^{1}$ Since the clinical symptoms of early-stage ovarian cancer are nontypical, nearly $70 \%$ patients are diagnosed at an advanced stage (III and IV). According to the National Comprehensive Cancer Network (NCCN) guidelines for advanced and metastatic ovarian cancer, platinum-based chemotherapy following debulking surgery is the standard regimen. ${ }^{2}$ Nevertheless, the recurrence rate is $70-80 \%$ and subsequent line therapies are needed for relapsed patients. Furthermore, therapeutic strategies after recurrence are limited and the 5-year overall survival rate is $40-50 \% .{ }^{3,4}$ Considering unsatisfactory therapeutic outcomes, it is imperative to explore more favorable treatments.

In recent years, targeted therapies have been a highlight in studies of ovarian cancer. ${ }^{5-8}$ Angiogenesis plays an essential important role in tumor growth, proliferation, progression and metastasis. ${ }^{9}$ The VEGF/VEGFR signaling pathway is the most important regulatory pathway inducing tumor angiogenesis. ${ }^{10}$ The VEGF family proteins include VEGF-A, VEGF-B, VEGF-C, VEGF-D and placental growth factor (PlGF) and bind three different tyrosine kinase receptors of VEGFR1, VEGFR2 and VEGFR3. ${ }^{11,12}$ Among them, VEGFR2 is the major mediator and plays vital roles in the regulation of cell mitosis, angiogenesis and VEGF
Correspondence: Qisen Guo Shandong Academy of Medical Sciences, Shandong Cancer Hospital and Institute, Shandong Cancer Hospital affiliated to Shandong University, 440 Jiyan Road, Jinan, Shandong 250I I7, People's Republic of China

Email13|56|52085@I63.com 
diffusion. ${ }^{13,14}$ Increasing numbers of studies have demonstrated the roles of anti-VEGF antibodies and VEGFR inhibitors in the treatment of ovarian cancer.

Apatinib is a novel oral small molecule tyrosine kinase inhibitor that highly selectively targets VEGFR-2. It could block the migration and proliferation of VEGFR-induced endothelial cells and reduce the tumor microvascular density. Apatinib has shown favorable efficacy as a subsequent-line treatment for advanced or metastatic chemo-resistant gastric cancer and has been approved by the Food and Drug Administration of the People's Republic of China. ${ }^{15-19}$ Furthermore, apatinib has demonstrated encouraging anti-tumor activities and tolerable toxicities in several solid tumors, including lung carcinomas, breast cancer, hepatocellular carcinoma and osteosarcoma cancers, among other tumor. ${ }^{20,21}$

Up to now, there has been limited data on the clinical efficacy and toxicity of apatinib monotherapy for ovarian cancer. Herein, we report a patient with ovarian cancer who was treated with apatinib following failure of the multi-line therapy and achieved a good clinical efficacy.

\section{Case report}

In August 2012, a 47-year-old woman discovered a pelvic mass by a physical examination, and then underwent cytoreductive surgery. Postoperative pathological confirmed that it was a bilateral ovarian serous papillary cystadenocarcinoma, poorly differentiated. A malignant nodule was found on the left side of the primary tumor. A total of eight lymph nodes on parametrium, omentum, epityphlon, bilateral iliac para-vessels and bilateral pelvic were biopsied and no tumor infiltration was found. Postoperative staging was pT1cN0M0, Ic. The patient received six cycles of first-line chemotherapy with TC (paclitaxel $120 \mathrm{mg} \mathrm{d} 1, \mathrm{~d} 8+$ carboplatin $0.5 \mathrm{~g} \mathrm{~d} 2$ in every 28 days) regimen. After twenty months (In September 2014), positron emission tomography-computed tomography (PETCT) showed multiple metastases, including hepatic and incision implantation metastasis. GP regimen (gemcitabine + cisplatin) (a specific treatment is not clear) was then administered for six cycles as a second-line therapy.

In March 2015, the patient came to our hospital for the first time. Abdominal computed tomography (CT) showed progression of hepatic metastases (Figure 1A1-A3). One cycle of DC/CIK + docetaxel regimen followed by one cycle of DP regimen (docetaxel $120 \mathrm{mg} \mathrm{d} 1+$ oxaliplatin $200 \mathrm{mg} \mathrm{d} 2$ ) and two cycles of TP regimen (liposome paclitaxel $210 \mathrm{mg} \mathrm{d} 1+$ lobaplatin $50 \mathrm{mg} \mathrm{d}$ ) was administrated, but the disease progressed continuously. Then she received six cycles of PP regimen (pemetrexed $0.8 \mathrm{~g} \mathrm{~d} 1+$ nedaplatin $120 \mathrm{mg} \mathrm{d} 1$ ) as subsequent therapy. In February 2016, the patient received targeted therapy with icotinib at home without doctors' permission. After four months, the CT scan showed new metastatic lesions in the pelvic peritoneal, omentum and glisson's capsule, and retroperitoneal lymph node metastases. Besides, hepatic and incision implantation metastasis lesions were also enlarged (Figure 1B1, B2, C1, C2). The CA125 level increased to $328.40 \mathrm{U} / \mathrm{mL}$. The therapeutic evaluation indicated PD and the clinical stage was stage IV (rT0N1M1). From June 2016, four cycles IF regimen (irinotecan $120 \mathrm{mg}$ $\mathrm{d} 1+$ capecitabine $80 \mathrm{mg} \mathrm{d} 8$ ) were administered. The response after two cycles was stable disease (SD) but went PD after four cycles (Figure 2A1, B1). The CA125 level was $408.9 \mathrm{U} / \mathrm{mL}$ and the CA15-3 level was137.5 U/mL.

Considering the patient had a good performance status, apatinib (250 mg p.o. daily) was administered as the subsequent-line treatment from October 2016. Blood pressure, liver function, kidney function and blood routine were tested regularly during the administration. After two months of apatinib treatment, the first therapeutic evaluation was stable disease (SD) (Figure 2 A2, B2). The CA19-9 level and the CA15-3 level showed a downward trend after the first rise (Figure 3).

Similar CT scan results were achieved after four months treatment (Figure 2 A3, B3). At the same time, the CA125 level decreased significantly from $563.2 \mathrm{U} / \mathrm{mL}$ to $131.6 \mathrm{U} / \mathrm{mL}$, and the CA15-3 level also declined sharply from $245.9 \mathrm{U} / \mathrm{mL}$ to $64.12 \mathrm{U} / \mathrm{mL}$. After eight months treatment (In June 2017), the level of CA125 and CA15-3 went up again (Figure 3), but the therapeutic evaluation on tumor volume exhibited SD. From August 9, 2017, to August 8, 2018, the CT scan showed no obvious changes (Figure 2 A4-A6, B4-B6), the CA125 level was maintained between $158.1 \mathrm{U} / \mathrm{mL}$ and $229.2 \mathrm{U} / \mathrm{mL}$, the CA15-3 level was maintained between $88.0 \mathrm{U} / \mathrm{mL}$ and 107.9 U/mL (Figure 3). In October 2018, the PET-CT showed disease progression (Figure 4). The level of CA125 and CA153 also increased. The therapeutic evaluation was PD and progression-free survival (PFS) for apatinib monotherapy was 24 months. The patient is still alive.

During the apatinib treatment, the main treatmentrelated adverse events (AEs) were Grade 1 hypertension and Grade 1 hand-foot syndrome according to the Common Terminology Criteria for Adverse Events (CTCAE) (Version 4.0). All side effects were tolerable and well managed. 

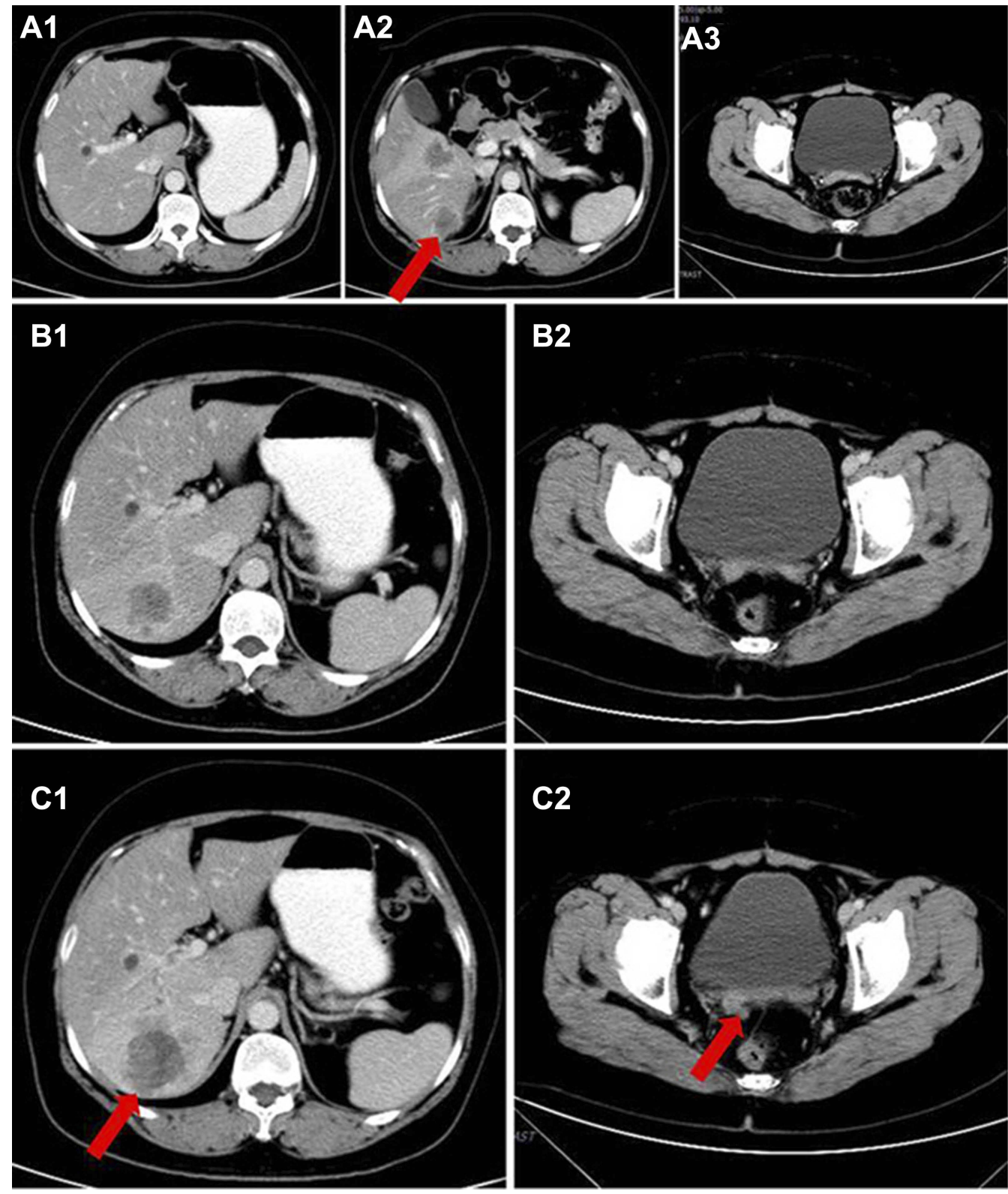

Figure I Hepatic metastases and incision implantation metastasis before apatinib treatment. Metastatic lesions when the patient first attended our hospital on March 2015 (AI, A2, A3); During the treatment of icotinib, the metastatic mass became bigger (CI, C2, June 2016), compared to two months earlier (B I, B2, April 2016). Red arrows indicate the hepatic metastasis and incision implantation metastasis.

\section{Discussion}

Ovarian cancer accounts for $2.5 \%$ of all malignancies among female but $5 \%$ of female cancer death, which pose a threat to the life of female patients. ${ }^{1}$ Because of the high relapse rate and poor prognosis of advanced ovarian cancer, new effective regimens are urgently needed. Currently, targeted therapies for ovarian cancer have produced significant breakthroughs, including antiangiogenic agents, poly (ADP-ribose) polymerase inhibitors and signaling pathway inhibitors. ${ }^{22}$ Among them, antiangiogenic agents have the potential advantages of low toxicity and less acquired resistance in cancer treatment. $^{23,24}$

Bevacizumab, a humanized monoclonal antibody targeting VEGF, was the first targeted antiangiogenic agent approved for ovarian cancer by the American Food and Drug Administration. ${ }^{25,26}$ The combination therapy of bevacizumab and chemotherapy was approved by the European Medicines Agency for the first-line treatment of advanced epithelial ovarian cancer and for the treatment of recurrent platinum- 

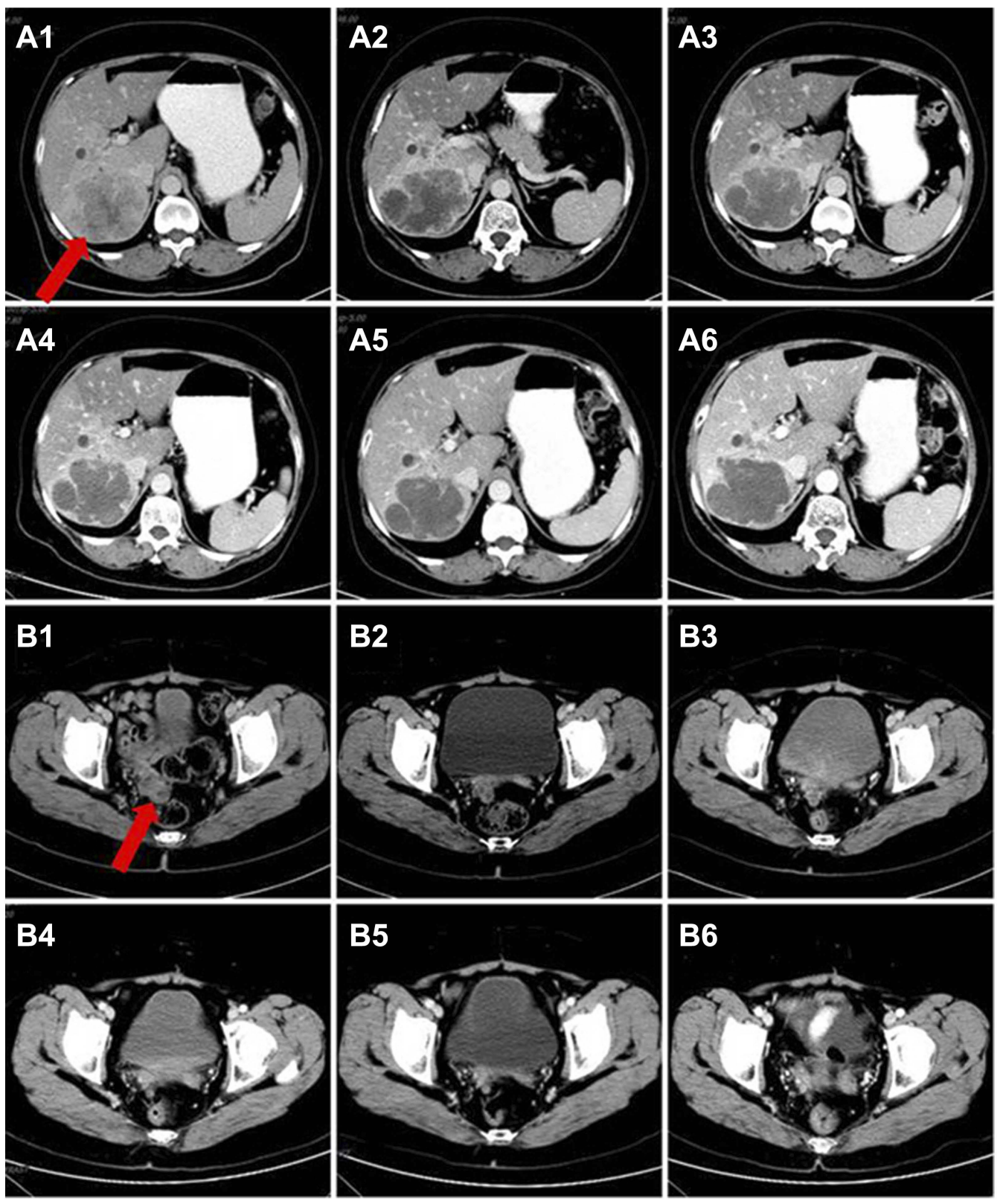

Figure 2 Hepatic metastasis and incision implantation metastasis change during apatinib treatment. Hepatic metastatic mass and incision implantation metastasis lesion before the treatment of apatinib (AI, BI, October 2016) and after two months of apatinib treatment (A2, B2); From March 20I7 to August 20I8, the metastatic lesions showed no obvious change (A3-A6, B3-B6). Red arrows indicate the hepatic metastasis and incision implantation metastasis.

sensitive epithelial ovarian cancer. ${ }^{25,27}$ Antiangiogenic agents targeting VEGFR (such as cediranib, pazopanib, nintedanib and sorafenib) have also been frequently investigated for ovarian cancer in clinical trials, ${ }^{28}$ but none of them are available in mainland China ${ }^{29-33}$ (Table1). The NCCN panel encourages to participate in ongoing clinical trials, in order to investigate the role of anti-angiogenesis agents in the treatment of ovarian cancer, both in primary disease and recurrence settings. $^{2}$
Apatinib, also known as YN968D1, is a potent VEGFR2 tyrosine kinase inhibitor that can inhibit the proliferation, migration and angiogenesis of vascular endothelial cells and can circumvent cancer cell resistance to other antineoplastic agent. $^{34}$ By specifically binding to VEGFR-2, apatinib blocks VEGF binding and subsequent VEGFR-2 autophosphorylation and inhibits downstream phosphorylation of extracellular signal-regulated kinases. ${ }^{20,35}$ Compared with bevacizumab, apatinib is cheaper and easier to 


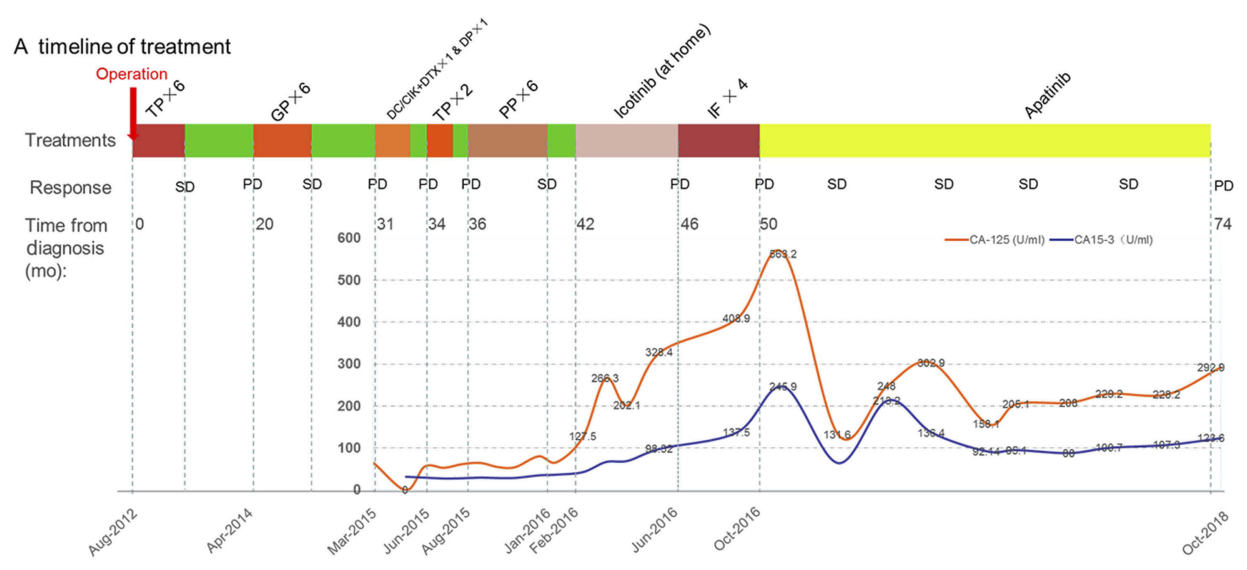

Figure 3 Timeline of treatment and trend in level of CA 125 and CA 15-3 during treatment. Green color indicates time without treatment.

Abbreviations: TC, paclitaxel/carboplatin; GP, gemcitabine/cisplatin; DC/CIK, dendritic cell/cytokine induced killer biological cell immunotherapy; DTX, docetaxel; TP, liposome paclitaxel/lobaplatin; PP, pemetrexed/nedaplatin; IF, irinotecan/capecitabine; SD, stable disease; PD, progressive disease.

administer. ${ }^{36}$ Phase I-III clinical trials showed that apatinib improved PFS and overall survival (OS) in patients with advanced gastric or gastroesophageal junction adenocarcinoma for whom standard chemotherapy had failed. Furthermore, apatinib has demonstrated encouraging antitumor activities and manageable toxicities in multiple solid tumors, both as a monotherapy and in combination with other drugs. ${ }^{16,37-40}$

There are already several explorations about the effect of apatinib in ovarian cancer. Mingming Miao et al conducted a Phase II to investigate effect apatinib monotherapy $(250 \mathrm{mg} /$ day $)$ for patients with recurrent, platinumresistant, pre-treated epithelial ovarian cancer. A total of 29 patients were included in this study and 28 patients were eligible for response evaluations. No complete response was observed, 12 patients achieved partial response and 8 patients experienced SD, with obejective response rate (ORR) and disease control rate (DCR) of $41.4 \% \quad(95 \%$ CI, 23.3-59.4) and $68.9 \% \quad(95 \% \quad \mathrm{CI}$, 52.1-85.8). 17 patients were still alive at the last followup. The median PFS was 5.1 months (95\% CI, 3.8 months-6.5 months) and median OS was 14.5 months (95\% CI, 12.4 months-16.4 months), respectively. Regarding safety of apatinib, the main treatment-related AEs were hand-foot syndrome (51.7\%), hypertension (34.6\%), nausea and vomiting (31.0\%). Most of the AEs were mild and tolerated, with $58.6 \%$ of grade 1 and grade 2 AEs and no grade 4 AEs. ${ }^{40}$ Chun Yan Lan et al evaluated the efficacy and toxicity of apatinib $(500 \mathrm{mg} /$ day) combined with etoposide $(50 \mathrm{mg} /$ day) in patients with platinum-resistant or platinum-refractory recurrent ovarian cancer. The results showed favorable efficacy of an oral combination, with an mPFS of 8.1 months (95\% CI, 2.8 months-13.4 months). In the intention-to-treat and perprotocol populations, the ORRs were 54\% (95\% CI, 36.6-71.2) and 61\% (95\% CI, 42.2-78.2), respectively. In their study, the toxicities of apatinib were controllable. Common AEs included hypertension (32\%), proteinuria (41\%) and hand-foot syndrome (29\%), similar to previously reported studies. ${ }^{41}$

In the present case, the patient started to receive apatinib monotherapy after failure of almost all considerable regimens. Extraordinarily, the mass lesions were well controlled and the comprehensive therapeutic evaluation constantly indicated SD. The PFS of this patient was up to 24 months, which is much longer than ever reported cases with apatinib monotherapy. According to the research of Scott et al, apatinib could reverse the P-glycoprotein $\mathrm{ABCB} 1$-and $\mathrm{ABCG} 2$-mediated multidrug resistance in drug-resistant solid tumor cells by inhibiting their transport function, so as to circumvents cancer cell resistance to other anti-tumor drugs. ${ }^{20}$ In addition, apatinib can also inhibit JAK/STAT3 and PI3K/Akt signaling pathways, and subsequently inhibit ovarian cancer cell motility, invasion and EMT. ${ }^{42}$ The deeper mechanism underlying the effects of apatinib on ovarian cancer remains unclear, which still warrants further investigation.

Although this is an individual case, apatinib monotherapy exhibited its efficacy in patient with advanced ovarian cancer. However, there are still several unclear points. Firstly, the level of CA125 and CA15-3 of this patient presented a double peak curve after apatinib monotherapy. It has been demonstrated that the decrease of the CA125 level predicts good prognosis. ${ }^{43,44}$ Whether there is an 

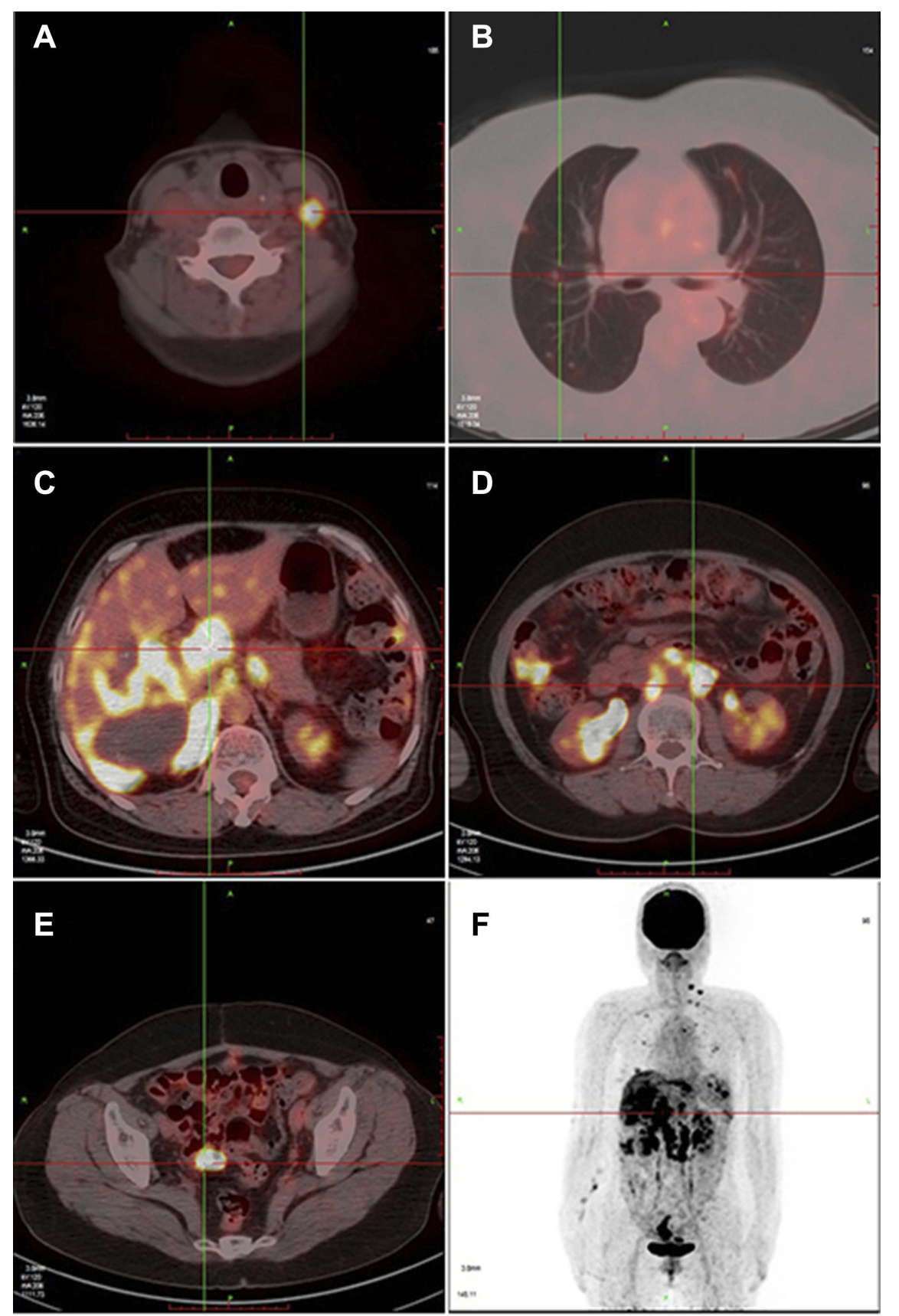

Figure 4 Positron emission tomography-computed tomography scan showed that the disease had progressed, metastatic lesions in liver, lung and the pelvic peritoneal had progressed and multiple lymph node metastases (include left lower neck, left superior clavicle, left internal breast area, anterior costal diaphragm angle, abdominal cavity and retroperitoneum) (A-F).

intrinsic link between this phenomenon and long-term stable state of disease deserves further discussion. Secondly, it has been previously reported that some toxicities could be predictive biomarkers for the good treatment efficacy of VEGFR tyrosine kinase inhibitors. ${ }^{45}$ In the AEROC study, exploratory analysis showed that handfoot syndrome might be related to improved PFS. ${ }^{41}$
Xinyang Liu et al found that the presence of hypertension and hand-foot syndrome in the first four weeks was associated with increased mOS, mPFS and DCR. ${ }^{46}$ This case was in line with this conclusion. Whether similar results could be found in ovarian cancer still needs further validation. Finally, what is the appropriate dose and best time to use apatinib in ovarian cancer patients? To answer these 


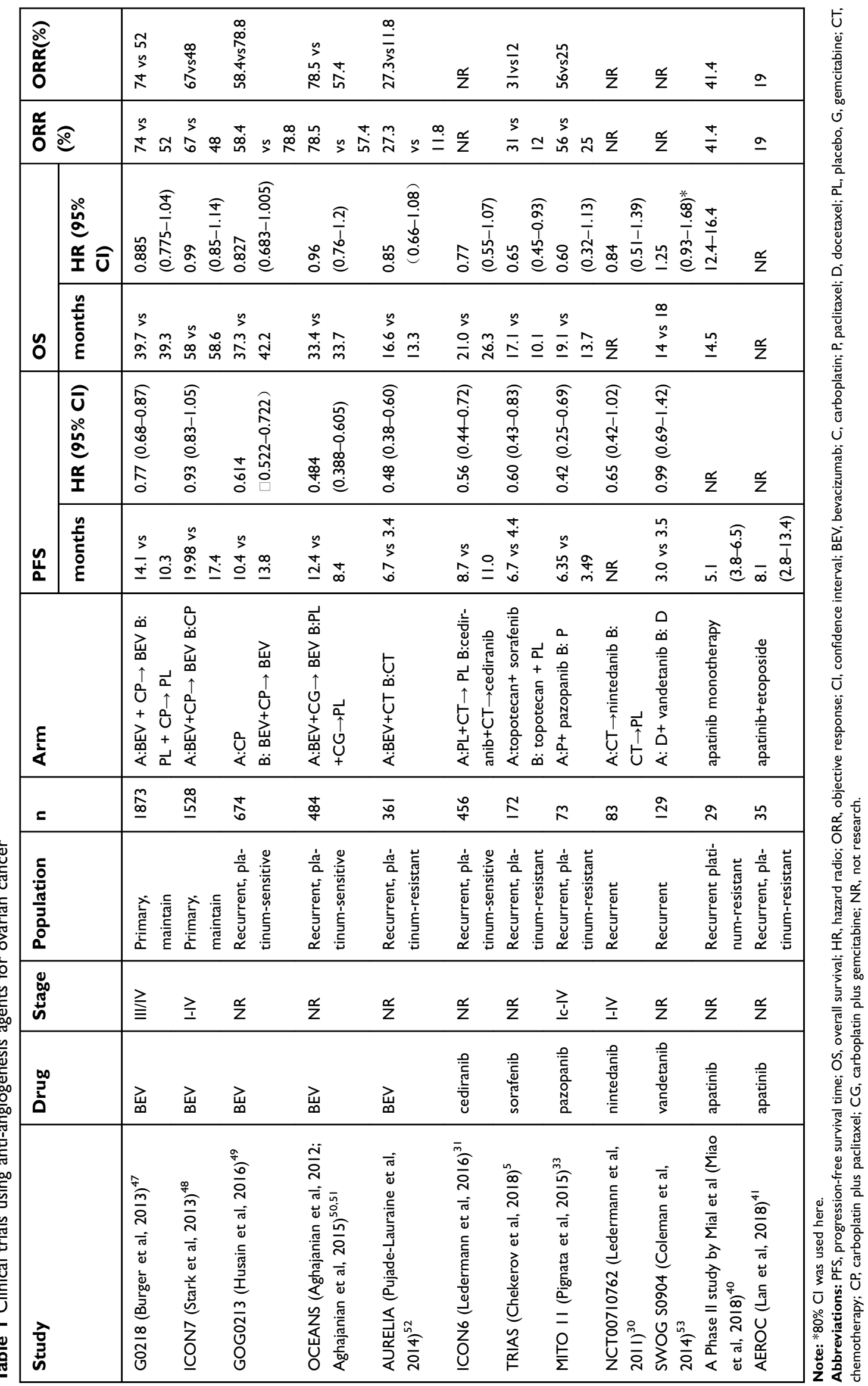


questions, more clinical studies are needed; and furthermore, these questions could offer guidelines for clinical treatment.

\section{Conclusion}

We reported a patient with relapsed ovarian cancer who had experienced extraordinary response to apatinib after failed multi-line therapy. This case indicated that apatinib could be a feasible option for patients with advanced ovarian cancer. However, as a single case report, it also has some limitations. Therefore, further observation and exploration are needed to validate its efficacy and safety in the treatment of ovarian cancer.

\section{Ethical approval}

The patient provided written informed consent for the case details and images to be published. All procedures performed in studies involving human participants were in accordance with the ethical standards of the institutional and/or national research committee and with the 1964 Helsinki declaration and its later amendments or comparable ethical standards.

\section{Acknowledgments}

The authors thank the patient for her participation and her agreement to publication of the report. No funding was received for this study.

\section{Disclosure}

The authors report no conflicts of interest in this work.

\section{References}

1. Torre LA, Trabert B, DeSantis CE, et al. Ovarian cancer statistics, 2018. CA Cancer J Clin. 2018;68(4):284-296. doi:10.3322/caac.21456

2. Morgan RJ Jr., Armstrong DK, Alvarez RD, et al. Ovarian cancer, version 1.2016, NCCN clinical practice guidelines in oncology. J National Compr Cancer Network. 2016;14(9):1134-1163.

3. Cheng Y, Zhang J, Geng H, Qin S, Hua H. Multiline treatment combining apatinib with toptecan for platinum-resistant recurrent ovarian cancer patients: a report of three cases. Onco Targets Ther. 2018;11:1989-1995. doi:10.2147/OTT.S158141

4. Liu D, Ha C, Zhang X, Zhang Z, Liu P. Molecular implication of ADAM-15 and -17 in intrauterine adhesions. Eur J Obstet Gynecol Reprod Biol. 2013;170(1):264-269. doi:10.1016/j.ejogrb.2013.06.036

5. Chekerov R, Hilpert F, Mahner S, et al. Sorafenib plus topotecan versus placebo plus topotecan for platinum-resistant ovarian cancer (TRIAS): a multicentre, randomised, double-blind, placebo-controlled, phase 2 trial. Lancet Oncol. 2018. doi:10.1016/S1470-2045(18)30372-3

6. Stewart J, George A, Banerjee S. Olaparib for the treatment of relapsed ovarian cancer with a BRCA1/2 mutation. Expert Rev Anticancer Ther. 2018;18(10):947-958. doi: 10.1080/14737140. 2018.1510323
7. Veskimae K, Scaravilli M, Niininen W, et al. Expression analysis of platinum sensitive and resistant epithelial ovarian cancer patient samples reveals new candidates for targeted therapies. Transl Oncol. 2018;11(5):1160-1170. doi:10.1016/j.tranon.2018.07.010

8. Vetter MH, Hays JL. Use of targeted therapeutics in epithelial ovarian cancer: a review of current literature and future directions. Clin Ther. 2018;40(3):361-371. doi:10.1016/j.clinthera.2018.01.012

9. Hanahan D, Weinberg RA. Hallmarks of cancer: the next generation. Cell. 2011;144(5):646-674. doi:10.1016/j.cell.2011.02.013

10. Koch S, Tugues S, Li X, Gualandi L, Claesson-Welsh L. Signal transduction by vascular endothelial growth factor receptors. Biochem J. 2011;437(2):169-183. doi:10.1042/BJ20110301

11. Olsson AK, Dimberg A, Kreuger J, Claesson-Welsh L. VEGF receptor signalling - in control of vascular function. Nat Rev Mol Cell Biol. 2006;7(5):359-371. doi:10.1038/nrm1911

12. Sullivan LA, Brekken RA. The VEGF family in cancer and antibody-based strategies for their inhibition. mAbs. 2010;2 (2):165-175.

13. Ferrara N. Role of vascular endothelial growth factor in regulation of physiological angiogenesis. Am J Physiol Cell Physiol. 2001;280(6): C1358-1366. doi:10.1152/ajpcell.2001.280.6.C1358

14. Lignet F, Benzekry S, Wilson S, et al. Theoretical investigation of the efficacy of antiangiogenic drugs combined to chemotherapy in xenografted mice. J Theor Biol. 2013;320:86-99. doi:10.1016/j. jtbi.2012.12.013

15. Geng R, Li J. Apatinib for the treatment of gastric cancer. Expert Opin Pharmacother. 2015;16(1):117-122. doi:10.1517/ 14656566.2015.981526

16. Ruan H, Dong J, Zhou X, et al. Multicenter phase II study of apatinib treatment for metastatic gastric cancer after failure of second-line chemotherapy. Oncotarget. 2017;8(61):104552-104559. doi:10.18632/oncotarget.21053

17. Tian S, Quan H, Xie C, et al. YN968D1 is a novel and selective inhibitor of vascular endothelial growth factor receptor-2 tyrosine kinase with potent activity in vitro and in vivo. Cancer Sci. 2011;102(7):1374-1380. doi:10.1111/j.1349-7006.2011.01939.x

18. Li J, Qin S, Xu J, et al. Apatinib for chemotherapy-refractory advanced metastatic gastric cancer: results from a randomized, placebo-controlled, parallel-arm, phase II trial. $J$ Clin Oncol. 2013;31(26):3219-3225. doi:10.1200/JCO.2013.48.8585

19. Li J, Qin S, Xu J, et al. Randomized, double-blind, placebo-controlled phase iii trial of apatinib in patients with chemotherapy-refractory advanced or metastatic adenocarcinoma of the stomach or gastroesophageal junction. J Clin Oncol. 2016;34 (13):1448-1454. doi:10.1200/JCO.2015.63.5995

20. Scott AJ, Messersmith WA, Jimeno A. Apatinib: a promising oral antiangiogenic agent in the treatment of multiple solid tumors. Drugs Today. 2015;51(4):223-229. doi:10.1358/dot.2015.51.4.2320599

21. Zhao D, Hou H, Zhang X. Progress in the treatment of solid tumors with apatinib: a systematic review. Onco Targets Ther. 2018;11:4137-4147. doi:10.2147/OTT.S172305

22. Chase DM, Chaplin DJ, Monk BJ. The development and use of vascular targeted therapy in ovarian cancer. Gynecol Oncol. 2017;145(2):393-406. doi:10.1016/j.ygyno.2017.01.031

23. Fontanella C, Ongaro E, Bolzonello S, Guardascione M, Fasola G, Aprile G. Clinical advances in the development of novel VEGFR2 inhibitors. Ann transl med. 2014;2(12):123.

24. Teoh DG, Secord AA. Antiangiogenic therapies in epithelial ovarian cancer. Cancer Control. 2011;18(1):31-43. doi:10.1177/ 107327481101800105

25. Dhillon S. Bevacizumab combination therapy: a review of its use in patients with epithelial ovarian, fallopian tube, or primary peritoneal cancer. BioDrugs. 2013;27(4):375-392. doi:10.1007/s40259-013$0043-4$ 
26. Ferrara N, Hillan KJ, Gerber HP, Novotny W. Discovery and development of bevacizumab, an anti-VEGF antibody for treating cancer. Nat Rev Drug Discovery. 2004;3(5):391-400. doi:10.1038/ $\operatorname{nrd} 1381$

27. Della Pepa C, Banerjee S. Bevacizumab in combination with chemotherapy in platinum-sensitive ovarian cancer. Onco Targets Ther. 2014;7:1025-1032. doi:10.2147/OTT.S40527

28. Ntanasis-Stathopoulos I, Fotopoulos G, Tzanninis IG, Kotteas EA. The emerging role of tyrosine kinase inhibitors in ovarian cancer treatment: a systematic review. Cancer Invest. 2016;34(7):313-339. doi:10.1080/07357907.2016.1206117

29. Du Bois A, Kristensen G, Ray-Coquard I, et al. Standard first-line chemotherapy with or without nintedanib for advanced ovarian cancer (AGO-OVAR 12): a randomised, double-blind, placebo-controlled phase 3 trial. Lancet Oncol. 2016;17(1):78-89. doi:10.1016/S1470-2045(15)00366-6

30. Ledermann JA, Hackshaw A, Kaye S, et al. Randomized phase II placebo-controlled trial of maintenance therapy using the oral triple angiokinase inhibitor BIBF 1120 after chemotherapy for relapsed ovarian cancer. J Clin Oncol. 2011;29(28):3798-3804. doi:10.1200/ JCO.2010.33.5208

31. Ledermann JA, Embleton AC, Raja F, et al. Cediranib in patients with relapsed platinum-sensitive ovarian cancer (ICON6): a randomised, double-blind, placebo-controlled phase 3 trial. Lancet. 2016;387 (10023):1066-1074. doi:10.1016/S0140-6736(15)01167-8

32. Friedlander M, Hancock KC, Rischin D, et al. A phase II, open-label study evaluating pazopanib in patients with recurrent ovarian cancer. Gynecol Oncol. 2010;119(1):32-37. doi:10.1016/j. ygyno.2010.05.033

33. Pignata S, Lorusso D, Scambia G, et al. Pazopanib plus weekly paclitaxel versus weekly paclitaxel alone for platinum-resistant or platinum-refractory advanced ovarian cancer (MITO 11): a randomised, open-label, phase 2 trial. Lancet Oncol. 2015;16 (5):561-568. doi:10.1016/S1470-2045(15)70115-4

34. Mi YJ, Liang YJ, Huang HB, et al. Apatinib (YN968D1) reverses multidrug resistance by inhibiting the efflux function of multiple ATP-binding cassette transporters. Cancer Res. 2010;70 (20):7981-7991. doi:10.1158/0008-5472.CAN-10-0111

35. Zhang H. Apatinib for molecular targeted therapy in tumor. Drug Des Devel Ther. 2015;9:6075-6081. doi:10.2147/DDDT.S97235

36. Li CM, Liu ZC, Bao YT, Sun XD, Wang LL. Extraordinary response of metastatic pancreatic cancer to apatinib after failed chemotherapy: a case report and literature review. World J Gastroenterol. 2017;23 (41):7478-7488. doi:10.3748/wjg.v23.i41.7478

37. Hu X, Cao J, Hu W, et al. Multicenter phase II study of apatinib in non-triple-negative metastatic breast cancer. BMC Cancer. 2014;14:820. doi:10.1186/1471-2407-14-820

38. Tang J, Li XY, Liang JB, Wu PL, Li X. Apatinib plus chemotherapy shows clinical activity in advanced NSCLC: a retrospective study. Oncol Res. 2018. doi:10.3727/096504018X15288447760357

39. Wang L, Liang L, Yang T, et al. A pilot clinical study of apatinib plus irinotecan in patients with recurrent high-grade glioma: clinical trial/ experimental study. Medicine. 2017;96(49):e9053. doi:10.1097/ MD.0000000000009053

40. Miao M, Deng G, Luo S, et al. A phase II study of apatinib in patients with recurrent epithelial ovarian cancer. Gynecol Oncol. 2018;148(2):286-290. doi:10.1016/j.ygyno.2017.12.013
41. Lan CY, Wang Y, Xiong Y, et al. Apatinib combined with oral etoposide in patients with platinum-resistant or platinum-refractory ovarian cancer (AEROC): a phase 2, single-arm, prospective study. Lancet Oncol. 2018;19(9):1239-1246. doi:10.1016/S1470-2045(18)30349-8

42. Ding J, Cheng XY, Liu S, et al. Apatinib exerts anti-tumour effects on ovarian cancer cells. Gynecol Oncol. 2019. doi:10.1016/j. ygyno.2019.01.010

43. Lukacsko I, Hernadi Z, Sapy T, Borsos A. The prognostic value of CA-125 in epithelial ovarian cancer patients during and after chemotherapy. Acta Chir Hung. 1997;36(1-4):213-214.

44. Moon JH, Lee HJ, Kang WD, Kim CH, Choi HS, Kim SM. Prognostic value of serum CA-125 in patients with advanced epithelial ovarian cancer followed by complete remission after adjuvant chemotherapy. Obstetrics Gynecology Sci. 2013;56(1):29-35. doi:10.5468/OGS.2013.56.1.29

45. Fan M, Zhang J, Wang Z, et al. Phosphorylated VEGFR2 and hypertension: potential biomarkers to indicate VEGF-dependency of advanced breast cancer in anti-angiogenic therapy. Breast Cancer Res Treat. 2014;143(1):141-151. doi:10.1007/s10549013-2793-6

46. Liu X, Qin S, Wang Z, et al. Early presence of anti-angiogenesis-related adverse events as a potential biomarker of antitumor efficacy in metastatic gastric cancer patients treated with apatinib: a cohort study. J Hematol Oncol. 2017;10(1):153. doi:10.1186/s13045-017-0521-0

47. Burger RA, Brady MF, Rhee J, et al. Independent radiologic review of the Gynecologic Oncology Group Study 0218, a phase III trial of bevacizumab in the primary treatment of advanced epithelial ovarian, primary peritoneal, or fallopian tube cancer. Gynecol Oncol. 2013;131:21-26. doi:10.1016/j.ygyno.2013.07.100

48. Stark D, Nankivell M, Pujade-Lauraine E, et al. Standard chemotherapy with or without bevacizumab in advanced ovarian cancer: quality-of-life outcomes from the International Collaboration on Ovarian Neoplasms (ICON7) phase 3 randomised trial.. Lancet Oncol. 2013;14:236-243. doi:10.1016/S1470-2045(12)70567-3

49. Husain A, Wang Y, Hanker LC, et al. Independent radiologic review of AURELIA, a phase 3 trial of bevacizumab plus chemotherapy for platinum-resistant recurrent ovarian cancer. Gynecol Oncol. 2016;142:465-470. doi:10.1016/j.ygyno.2016.05.011

50. Aghajanian C, Blank SV, Goff BA, et al. OCEANS: a randomized, double-blind, placebo-controlled phase III trial of chemotherapy with or without bevacizumab in patients with platinum-sensitive recurrent epithelial ovarian, primary peritoneal, or fallopian tube cancer. J Clin Oncol. 2012; 30:2039-2045. doi:10.1200/JCO.2012.42.0505

51. Aghajanian C, Goff B, Nycum LR, Wang YV, Husain A, Blank SV. Final overall survival and safety analysis of OCEANS, a phase 3 trial of chemotherapy with or without bevacizumab in patients with platinum-sensitive recurrent ovarian cancer. Gynecol Oncol. 2015;139:10-16. doi:10.1016/j.ygyno.2015.08.004

52. Pujade-Lauraine E, Hilpert F, Weber B, et al. Bevacizumab combined with chemotherapy for platinum-resistant recurrent ovarian cancer: The AURELIA open-label randomized phase III trial. J Clin Oncol. 2014;32:1302-1308. doi:10.1200/JCO.2013.51.4489

53. Coleman RL, Moon J, Sood AK, et al. Randomised phase II study of docetaxel plus vandetanib versus docetaxel followed by vandetanib in patients with persistent or recurrent epithelial ovarian, fallopian tube or primary peritoneal carcinoma: SWOG S0904. Eur J Cancer. 2014;50:1638-1648. doi:10.1016/j.ejca.2014.03.005 


\section{Publish your work in this journal}

OncoTargets and Therapy is an international, peer-reviewed, open access journal focusing on the pathological basis of all cancers, potential targets for therapy and treatment protocols employed to improve the management of cancer patients. The journal also focuses on the impact of management programs and new therapeutic agents and protocols on patient perspectives such as quality of life, adherence and satisfaction. The manuscript management system is completely online and includes a very quick and fair peer-review system, which is all easy to use. Visit http://www.dovepress.com/ testimonials.php to read real quotes from published authors. 